\title{
No evidence for effectiveness of interventions used to treat displaced permanent anterior teeth
}

\author{
Abstracted from \\ Belmonte FM, Macedo CR, Day PF, Saconato H, Fernandes Moça Trevisani V. \\ Interventions for treating traumatised permanent front teeth: luxated (dislodged) teeth. \\ Cochrane Database Syst Rev 2013; Issue 4. Art. No. CD006203. DOI: 10.1002/14651858.CD006203.pub2. \\ Address for correspondence: Luisa Fernandez Mauleffinch, Review Group Co-ordinator, \\ Cochrane Oral Health Group, MANDEC, School of Dentistry, University of Manchester, \\ Higher Cambridge Street, Manchester, M15 6FH, UK. E-mail: luisa.fernandez@manchester.ac.uk
}

\section{Question: What is the best way of managing displaced luxated permanent teeth?}

Data sources The Cochrane Oral Health Group Trials register, Cochrane Central Register of Controlled trials (CENTRAL), Medline, Embase, LILACS and Dissertation, Theses and Abstracts databases. Study selection Randomised controlled trials or quasi-randomised controlled trials that included a minimum follow-up period of 12 months. Data extraction and synthesis Two review authors independently and in duplicate assessed the eligibility of all reports identified in the searches. Authors were contacted for additional information where required.

Results No randomised or quasi-randomised controlled trials were found. Conclusions We found no randomised or quasi-randomised trials of interventions to treat displaced luxated permanent front teeth. Current clinical guidelines are based on available information from case series studies and expert opinions. Randomised controlled trials in this area of dental trauma are required to robustly identify the benefits of different treatment strategies.

\section{Commentary}

This systematic review addresses the effect of different interventions used in treatment of lateral, extrusive and intrusive luxations of permanent anterior teeth. Three databases and two trial registries were searched for randomised and quasi-randomised controlled trials. Reference lists were cross-checked for additional articles. Two review authors independently assessed articles for eligibility. Disagreements were referred to a third author and ultimately resolved by discussion with three authors. Three case series constituting more than 160 treated luxated teeth were identified but eventually excluded as they didn't meet the eligibility criteria.

Dental traumatology relies more on retrospective studies, case series and reports than randomised clinical trials. A search on Medline in the period from January 2001 to April 2012 revealed that nearly $30 \%$ of published articles in the Journal of Dental

This paper is based on a Cochrane Review published in the Cochrane Library 2013, issue 5 (see www.thecochranelibrary.com for information). Cochrane Reviews are regularly updated as new evidence emerges and in response to feedback, and the Cochrane Library should be consulted for the most recent version of the review.
Traumatology were case series and reports while randomised clinical trials constitute $2 \%$ only. ${ }^{1}$ This can be attributed to paucity of cases, absence of proper control group or the emergency nature of dental trauma.

The Cochrane review in hand is considered an 'empty review' with no studies eligible for inclusion. Empty reviews are of limited use for clinicians and decision makers as they encompass either no conclusion or conclusions based on excluded studies. ${ }^{2,3}$ Conclusions deducted from Cochrane systematic reviews rely - in most instances on randomised and quasi-randomised clinical trials, as they represent the top of the evidence pyramid. Subsequently, case series and reports are not included under the notion of 'low-level evidence' although they can influence clinical practice. ${ }^{4}$ Systematic reviews of case series and reports can provide an acceptable alternative to empty reviews as antecedent trials regarding treatment effectiveness can be identified and results can be presented either quantitatively ${ }^{1}$ or qualitatively. ${ }^{5}$ Further, researchers would possess a more solid background required for designing future randomised clinical trials. ${ }^{1}$

Although no conclusion could be drawn from this systematic review regarding treatment effectiveness, it highlights some methodological points to be considered for future relevant research such as; considering participants as the primary unit of analysis, full reporting of outcomes for each injured tooth in participants with multiple injured teeth, define primary outcome measures as tooth and pulp survival, mobility, pain and function, while secondary outcome measures to be considered are cost, patient satisfaction and quality of life.

\footnotetext{
Ahmed ElKhadem ${ }^{\mathbf{1}}$ and Passant Nagi ${ }^{2}$

${ }^{1}$ Evidence Based Dentistry Centre, Faculty of Oral and Dental Medicine, Cairo University, ${ }^{2}$ Pediatric Dentistry Department, Faculty of Oral and Dental Medicine, Cairo University, Egypt.

1. Elkhadem A, Mickan S, Richards D. Adverse events of surgical extrusion in treatment for crown-root and cervical root fractures: a systematic review of case series/reports. Dent Traumatol 2013; doi: 10.1111/edt.12051. [Epub ahead of print]

2. Lang A, Edwards N, Fleiszer A. Empty systematic reviews: hidden perils and lessons learned. J Clin Epidemiol 2007; 60: 595-597.

3. Yaffe J, Montgomery P, Hopewell S, Shepard LD. Empty reviews: a description and consideration of Cochrane systematic reviews with no included studies. PLoS One 2012; 7: e36626.

4. Chambers $\mathrm{D}$, Rodgers $\mathrm{M}$, Woolacott N. Not only randomized controlled trials, but also case series should be considered in systematic reviews of rapidly developing technologies. J Clin Epidemiol 2009; 62: 1253-1260.

5. Das B, Muthu MS. Surgical extrusion as a treatment option for crown-root fracture in permanent anterior teeth: a systematic review. Dent Traumatol 2013 doi: 10.1111/edt.12054. [Epub ahead of Printing]
} 